\title{
The Birth of Quark Stars: Photon-driven Supernovae?
}

\author{
Anbo Chen, Tianhong $\mathrm{Yu}$ and Renxin $\mathrm{Xu}$ \\ School of Physics, Peking University, Beijing 100871, China \\ r.x.xu@pku.edu.cn
}

\begin{abstract}
In this letter we propose a possible mechanism trying to alleviate the current difficulty in core-collapse supernovae by forming a strange quark star inside the collapsing core. Although the initial longtime cooling behavior of nascent strange stars is dominated by neutrino emissions, thermal emissions including photons and $e^{ \pm}$pair plasma do play a significant role in the explosion dynamics under this picture. The key to promote a successful shock outside a bare strange star is more likely to be the radiation pressure caused by thermal photons rather than neutrinos in conventional models. We observed through calculation that radiation pressure can push the overlying mantle away through photon-electron scattering with energy (the work done by radiation pressure) as much as $\sim 10^{51}$ erg if protoquark stars are born with temperatures higher than $\sim(30-40) \mathrm{MeV}$. This result not only indicates that strange quark stars should be bare ever since their formations, it could also provide a possible explanation to the formation of fireballs in cosmic long-soft $\gamma$-ray bursts associated to supernovae.
\end{abstract}

Subject headings: stars: supernovae: general - dense matter - pulsars: general stars: neutron

\section{Introduction}

Thanks to the advanced X-ray missions (e.g., Chandra and XMM-Newton), it is now high time for astrophysicists to research into the nature of pulsars and relevant issues. Pulsarlike stars are unique astro-laboratories to study matter at supranuclear density. On the one hand, due to the mathematical complexity of the nonlinear nature of quantum chromodynamics (QCD), one believed to be the underlying theory for strong interaction, we can not determine the state of supranuclear matter by first principles. Several speculations have to be presented in the literatures, including those currently focused state-of-the-art nuclear equations (normal neutron stars). Besides these conjectures, stable strange quark matter 
state (quark stars) has been alternatively proposed since quark matter is a direct consequence of the "asymptotic freedom" which was found experimentally in 1960s and proved by QCD in 1973. On the other hand, recent observations show new members of the family of pulsarlike stars (e.g., anomalous X-ray pulsars/soft gamma-ray repeaters, central compact objects, and dim thermal neutron stars), whose different manifestations are not well understood and could challenge the conventional scenario of normal neutron stars. In fact, neutron stars and quark stars should now be considered as two potential models equally possible for the nature of pulsar-like stars (see reviews e.g., Madsen 1999; Lattimer \& Prakash 2004; Glendenning 2000; Weber 2005; Xu 2006).

How do quark stars form? This is a question with bimodality of meaning. (i) It is straightforward to know their births if one believes pulsars are actually quark stars. The astrophysics of phase conversion from nuclear matter to quark matter during, e.g., spindown or accretion stages is investigated at a preliminary step (Ouved et al. 2002; Keränen et al. 2005); but that quark star formation occurs simultaneously during the collapsing of massive star's cores has not been treated previously, which will be focused in this paper. (ii) Could core-collapse-produced quark stars result in successful supernovae? While supernovae keep occurring above the sky, the failure to simulate an explosion successfully in calculations troubles astrophysicists over time. In reviewing the neutrino-driven explosion model, the call for an alternative mechanism grew stronger (Mezzacappa 2005; Buras et al. 2003). Since strange quark matter (SQM) could be the real ground state (Witten 1984), it is suggested that SQM-formation may help to overcome the energy difficulty in getting type-II supernovae successful, because more neutrinos should be radiated if phase-transition to SQM is included (Benvenuto \& Horvath 1989; Lugones et al. 1994; Dai et al. 1995; See a review of Horvath 2007).

However, it should be emphasized that bare quark surfaces could be essential to successful explosions, for both $\gamma$-ray bursts and core-collapse supernovae (Paczynski \& Haensel 2005; Xu 2005). The reason for that is simple and intuitive: due to the chromatic confinement, the photon luminosity of a quark surface is not limited by the Eddington limit. Regarding the ultra-high surface temperature of nascent strange stars (Haensel et al. 1991), we believe that the strong radiation pressure caused by enormous thermal emissions from strange stars might plays a more important role in promoting a shock, substituting neutrinos in conventional delayed shock mechanism. We call this proposed scenario as a photon-driven supernova. Quark stars formed in this way should be bare, and factually, there are possible observational evidence for bare strange stars (Xu et al. 1999; $\mathrm{Xu}$ 2002; Yue et al. 2006). 


\section{The Model}

Due to the different properties between neutron stars and strange stars, the consequent mechanism in explosion could be significantly altered. A detonation wave burning nuclear matter into strange matter spreads out from the core (Lugones et al. 1994), where the density declines as radius increases. A boundary of strange matter and nuclear matter will be set where the detonation wave stops when nuclear matter density drops blow a critical value. Nevertheless, the outer mantle on the other hand is still in-falling with hardly any variation since the detonation wave travels faster than the sound speed. In both the prompt and the delayed shock mechanisms, the previous shock which ejects the overlying mantle is initially generated by the bounce at the surface of neutron stars (Bethe 1990). However, if a nascent strange star is formed by a detonation wave, the strange matter behind the wavefront is considered nearly at rest (Lugones et al. 1994) and hence no bounce could be introduced.

As a consequence of the high optical opacity in the collapsing core with density $\rho$ and temperature $T$ (Padmanabhan 2000), $\kappa_{\mathrm{ph}}=\kappa_{\mathrm{ph}_{0}} \rho T^{3.5} \mathrm{~cm}^{2} \mathrm{~g}^{-1}$, the radiation pressure caused by photon-electron scattering prevents a thin layer of nuclear matters outside from falling onto the surface of nascent strange star and thus inverses its velocity, where $\kappa_{\mathrm{ph}_{0}}=$ $4 \times 10^{25} Z(1+X)$, atomic charge number $Z$, and hydrogen mass fraction $X$. This layer being affected extends to the sonic point because super-sonic fluid cannot be affected by perturbations downstream. As soon as the layer between the strange star surface and the sonic point assembles the radiation pressure after an equilibrium is established, a shock will be generated at the sonic point for the discontinuities in both density and pressure.

As the overlying mantle is driven outwards by the shock, a gap is left between the strange star and the out-going matter. This gap is filled up by a fireball, with high energy photons and electron-positron pair plasma emitted from the hot strange star. We illustrate the initial collapsing core divided into four parts in Fig. 1.

Though annihilation of $\gamma$ into $e^{+} e^{-}$pairs and the reverse process take place at such high energy scale, the total momentum and energy fluxes are preserved. Hence we may still adopt the formula $P_{\mathrm{rad}}=a T^{4} / 3$, where $P_{\mathrm{rad}}$ is radiation pressure and $a$ is the radiation constant. The pressure decreases as the fireball expands, with an assumption of $P_{\text {rad }} \propto r^{-n}$, where $r$ is the radius of fireball. In a radiation dominated relativistic fireball with a vacuum exterior, we have $n=4$, i.e., $P_{\text {rad }} \propto r^{-4}$ (Piran et al. 1993). However, the exterior of the fireball is actually not vacuum but fall-back matter, which must exert a force to the fireball. The expanding speed of fireball is lower than the speed of light, and the radiation pressure should then not drop so fast, i.e. the index $n$ could be smaller than 4 . In a special case for normal radiation, one may have $P_{\text {rad }} \propto r^{-2}$ from flux conservation. Therefore we generalize 
the relation as

$$
P_{\text {rad }}(r)=\frac{1}{3} a T^{4} \frac{R^{n}}{r^{n}},
$$

where $R$ is the radius of the strange star, and three cases of $n=4,3,2$ will be considered.

Our main goal is to check whether the work done on the overlying mantle by the radiation pressure is comparable to $10^{51} \mathrm{erg}$, the typical energy needed for a successful supernova explosion. The work done by the fireball reads,

$$
W=\int_{R}^{r_{\mathrm{f}}} 4 \pi r^{2} P_{\operatorname{rad}}(r) \mathrm{d} r .
$$

where $r_{\mathrm{f}}$ is the final distance that the radiation pressure could push the out layers to. The distance can be estimated by velocity of the shocked matter and the radiation-dominated timescale.

Since mean free path of photons in dense nuclear matter is extremely short, $\lambda=$ $1 /\left(\rho \kappa_{\mathrm{ph}}\right)=1 /\left(\kappa_{\mathrm{ph}_{0}} \rho^{2} T^{3.5}\right)$, radiative heating is very inefficient. The time scale for transferring most part of the energy to the shock is excessively shorter than the kinematic timescale, as we can see later in our calculation. The lack of energy transferred to the outside of fireball could essentially help a successful explosion. Moreover, we can therefore omit the thin layer that has been heated in region 2 and consider the pressure within these two regions contributed by degenerate electrons alone, complying with the polytropic equations: $P_{1} \propto \rho_{1}^{\gamma}, P_{2} \propto \rho_{2}^{\gamma}$. Because both the shock and the shocked matter are supported by the radiation pressure ultimately, we assume that pressure in region 2 equals to the radiation pressure for simplicity though in the actual explosion non-uniform pressure distribution must exists.

Meanwhile, we solve the relativistic shock equation (Landau \& Lifshitz 1999) and obtain the speed of the shocked matters moving outwards,

$$
\frac{v_{1}}{c}=\left[\frac{\left(P_{2}-P_{1}\right)\left(e_{2}+P_{1}\right)}{\left(e_{2}-e_{1}\right)\left(e_{1}+P_{2}\right)}\right]^{1 / 2}, \frac{v_{2}}{c}=\left[\frac{\left(P_{2}-P_{1}\right)\left(e_{1}+P_{2}\right)}{\left(e_{2}-e_{1}\right)\left(e_{2}+P_{1}\right)}\right]^{1 / 2} .
$$

where $v$ is velocity and $e$ stands for energy density. Note that these values are derived in a coordinate system where the shock wave surface is at rest. Subscript 1 and 2 represent corresponding values in regions 1 and 2 (defined in Fig. 1), respectively. Adopting the numerical solution of last good homology [Table 1 of Brown et al. (1982)] and applying the radiation pressure of Eq.(1) to region 2, the velocity of the shock's propagation rate can be achieved by

$$
V_{\text {shock }}=c^{2} \frac{v_{1}-V_{1}}{v_{1} V_{1}-c^{2}},
$$


where $V$ is the velocity in the inertial frame where the collapsing center is at rest. Thus the velocity of region 2 in the center inertial frame can be obtained,

$$
V_{2}=c^{2} \frac{V_{\text {shock }}+v_{2}}{V_{\text {shock }} v_{2}+c^{2}}=\frac{\mathrm{d} r}{\mathrm{~d} t} .
$$

Finally, we obtain the velocity of the shocked matter (about 0.1 speed of light) and hence the expansion speed of the fireball.

Strange quark matter at high density and small temperature is expected to exhibit color-superconductivity (CSC), induced by quark pairing and condensation at the Fermi surface, with energy gaps $\Delta \simeq 100 \mathrm{MeV}$ (Alford et al. 1998; Ouyed et al. 2005) and associated critical temperatures $T_{c} \simeq 0.6 \Delta$, above which thermal fluctuations destroy the condensate (Rajagopal \& Wilczek 2000). If the density is sufficiently high, a color-flavor locked (CFL) phase is the favored ground state (e.g., Ouyed et al. 2005).

The surface emissivity of photons with energies below $\hbar \omega_{\mathrm{p}} \simeq 23 \mathrm{MeV}\left(\omega_{\mathrm{p}}\right.$ : electromagnetic plasma frequency) is strongly suppressed (Usov 2001). As shown in Vogt et al. (2004), average photon energies in CFL matter at temperature $T$ are $\sim 3 T$. Therefore, when the surface temperature of the star drops below $T_{\mathrm{f}}=\hbar \omega_{\mathrm{p}} / 3 \simeq 7.7 \mathrm{MeV}$, the photon emissivity can be considered shut off. As long as we know the cooling behavior, we can then estimate the timescale of radiation-dominated period.

A nascent quark star is actually not an isothermal, homogeneous sphere (e.g., Ouyed et al. 2005). The thermal evolution is determined by the energy conservation and heat transport equations,

$$
C_{\mathrm{v}} \frac{\partial T}{\partial t}=-\frac{1}{r^{2}} \frac{\partial\left(r^{2} F_{r}\right)}{\partial r}-\epsilon_{\nu}, F_{r}=-\kappa \frac{\partial T}{\partial r}
$$

where $C_{\mathrm{v}}$ is the specific heat of the star matter, $\kappa$ is its thermal conductivity, $\epsilon$ is the neutrino emissivity, and $F_{r}$ is the heat flux at radius $r$.

For normal quark matter (Iwamoto 1982), one has

$$
C_{\mathrm{v}} \simeq 1.6 \times 10^{21} T_{9} \mathrm{erg} \mathrm{cm}^{-3} \mathrm{~K}^{-1}, \epsilon_{\nu}=3 \times 10^{24} T_{9}^{6} \mathrm{erg} \mathrm{cm}^{-3} \mathrm{~s}^{-1} .
$$

The heat capacity of the quark matter with color superconductivity (CFL phase, Blaschke et al. 2000) could be

$$
C_{\mathrm{v}} \simeq 5.1 \times 10^{21} T_{9}\left(T_{c} / T\right) \exp (-\Delta / T)\left[2.5-1.7 T / T_{c}+3.6\left(T / T_{c}\right)^{2}\right] \mathrm{erg} \mathrm{cm}^{-3} \mathrm{~K}^{-1},
$$

where $\Delta$ is the energy gap and $T_{c}$ is the critical temperature, and the neutrino emissivity for this CFL state is (Jaikumar \& Prakash 2001),

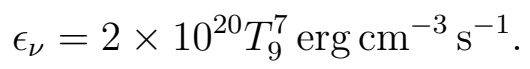


It is evident from Eq.(7) and Eq.(9) that the neutrino emissivity could be much lower in CFL state than in normal state of quark matter. Low $\nu$-emissivity favors keeping a high surface temperature of CFL stars (Fig. 2), that helps to form an energetic fireball. According to Shovkovy \& Ellis (2002), the conductivity in Eq.(6) reads

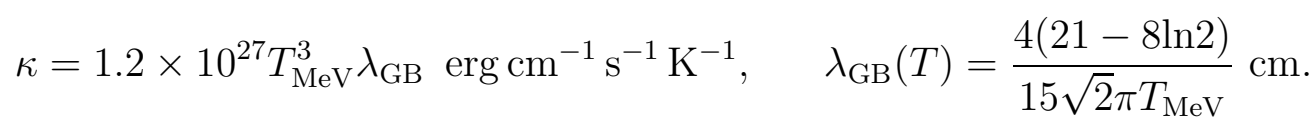

In addition, we choose the following boundary conditions: at the center and the surface of the star, we have $F(r=0)=0$ and $F(r=R)=\sigma T^{4}$. As for the initial condition, we assume the temperature of the star at $t=0$ be uniform, $T(t=0)=T_{0}$. The initial temperature of the new born strange star can be estimated to be tens of $\mathrm{MeV}$. Once we input $T_{0}$, Eq.(6) can be solved numerically. We can then obtain radiation-dominated timescale and hence calculate the total work done by the fireball.

\section{The Results}

In the calculation shown in Fig. 2, we find that the cooling rate of strange matter with CSC is slower than that of normal quark matter, and with bigger energy gap, the CSC matter cools faster. In about $\sim 1 \mathrm{~ms}$, the surface temperature of a CFL star will drop below 7.7 $\mathrm{MeV}$, the typical threshold of photon emission. The timescale of strong thermal radiation is adequately short, and thus we can safely use the assumption that the heating process in the thin layer in region 2 of Fig. 1 will not influence much to the mechanism.

In Fig. 3 we calculate the work done by radiation pressure with different pressure-radius relationships, i.e. different value of index $n$ in Eq.(1), and with different initial temperatures. It can be seen that, for a typical condition (e.g., $T_{0}=50 \mathrm{MeV}, R=10 \mathrm{~km}, n=3$ ), the work would exceed $2.7 \times 10^{51}$ erg within radiation dominated timescale, which may result in a successful explosion. For different initial temperatures, the work could pass $10^{51}$ erg once the initial temperature exceed about $40 \mathrm{MeV}$. Note that the initial temperature could be (30-40) MeV for normal neutron stars (Burrows \& Lattimer 1986). More energy (i.e., both gravitation and phase-transition energies) should be released for quark stars if the conjecture by Witten (1984) is correct. Unfortunately, it is still model-dependent to determine the real initial temperature of a quark star. From Fig.3, we could conclude that the total work done by the fireball could be enough for successful supernova if the initial temperatures is greater than $\sim(30-40) \mathrm{MeV}$. 


\section{Conclusions and Discussions}

A photon-driven mechanism is proposed for successful core-collapse supernovae, and bare strange quark stars are residues after the explosions. It is found through calculations that radiation pressure can push the overlying mantle away through photon-electron scattering with energy (the work done by radiation pressure) as much as $\sim 10^{51} \mathrm{erg}$, the typical energy needed for core-collapse supernovae.

Regarding the difference between nuclear matter and strange quark matter, a strange star can have a much higher surface temperature than a neutron star and accordingly a greater thermal photon emission. In addition, the strong electric field $\left(\sim 10^{17} \mathrm{~V} / \mathrm{cm}\right)$ on quark surface should play an important role in producing the thermal emission too [i.e., the Usov mechanism (Usov 1998, 2001)], which is in the same order of blackbody radiation when $T>5 \times 10^{10} \mathrm{~K}$. Furthermore, this distinction results in a huge radiation pressure that leads to a much faster explosion than the conventional delayed-shock model. The photon-driven supernova may benefit not only from the radiation pressure but also a much smaller photodissociation effect while most part of the mantle is blown away before the iron-cores could ever interact with high energy photons, hence making it possible to provide sufficient energy and promote a successful explosion.

Low-mass bare strange stars could form via accretion-induce collapse of white dwarfs (Xu 2005). A low energy budget is needed in this scenario since the gravitational binding energy of a white dwarf with approximate Chandrasekhar mass is only $\sim 10^{50} \mathrm{erg}$.

This photon-driven mechanism may also provide an alternative figure how a fireball can be produced during cosmic long-soft $\gamma$-ray bursts, which are observed associated with supernovae. It is worth noting that, due to the chromatic confinement of quark surfaces, the baryon contamination would be very low in such fireballs, that is necessary in the models (Cheng \& Dai 1996; Dai \& Lu 1998; Wang et al. 2000). Similar $\gamma$-ray burst fireballs due to the color-flavor locked phase-transition as well as magnetic field decay were noted (Ouyed et al. 2005, 2006). It is worth noting that Cui et. al. (2007) showed statistically that long-soft $\gamma$-ray burst could be really related to supernova and that the asymmetry of bursts associated with supernova would cause the kick of pulsars.

The authors would like to thank the referee for his/her very constructive comments and suggestions, and wish to acknowledge useful discussions with Guojun Qiao, Weiwei Zhu, Youling Yue and Moda Cao at Peking University. We are indebted to J. Horvath for many general discussions about supernova by forming bare quark stars. This work is supported by NSFC (10573002, 10778611), the Key Grant Project of Chinese Ministry of Education 
(305001), the program of the Light in China's Western Region (LCWR, No. LHXZ200602), and the Chun-Tsung Foundation.

\section{REFERENCES}

Alford, M., Rajagopal, K., \&Wilczek, F. 1998, Phys. Lett. B422, 247

Benvenuto, O. G., \& Horvath, J. E. 1989, Phys. Rev. Lett., 63, 716

Bethe, H. A. 1990, Rev. Mod. Phys., 62, 801

Blaschke, D., Klähn, T., \& Voskresensky, D. N. 2000, ApJ, 533, 406

Brown, G. E., Bethe, H. A., \& Baym, G. 1982, Nucl. Phys., A375, 481

Buras, R., Rampp, M., Janka, H.-Th., \& Kifonidis, K. 2003, Phys. Rev. Lett., 90, 241101

Burrows, A., Lattimer, J. M. 1986, ApJ, 307, 178

Cheng, K. S., Dai, Z. G. 1996, Phys. Rev. Lett. 77, 1210

Cui, X. H., Wang, H. G., Xu, R. X., Qiao, G. J. 2007, A\&A, 472, 1 (astro-ph/0701888)

Dai, Z. G., Lu, T. 1998, Phys. Rev. Lett. 81, 4301

Dai, Z. G., Peng, Q. H., \& Lu, T. 1995, ApJ, 440, 815

Glendenning, N. K. Compact Stars (Springer-Verlag, New York, 2000), 2nd ed.

Goldreich, P., \& Weber, S. V. 1980, ApJ, 238, 991

Haensel, P., Paczynski, B., \& Amsterdamski, P. 1991, ApJ, 375, 209

Horvath, J. E. 2007, eprint, astro-ph/0703233

Iwamoto, N. 1982, Ann. Phys., 141, 1

Jaikumar, P., \& Prakash, M. 2001, Phys. Lett. B, 516, 345

Keränen, P., Ouyed, R., \& Jaikumar, P. 2005, ApJ, 618, 485

Landau, L. D., \& Lifshitz, E. M. Fluid Mechanics (Butterworth-Heinemann, Oxford, 1999), 2nd ed.

Lattimer, J. M., \& Prakash, M. 2004, Science, 304, 536 
Lugones, G., Benvenuto, O. G., \& Vucetich, H. 1994, Phys. Rev. D, 50, 6100

Madsen, J. 1999, Lecture Notes in Physics, 516, 162 (astro-ph/9809032)

Mezzacappa, A. 2005, in: 1604-2004: Supernovae as Cosmological Lighthouses, ASPC, Vol.342, p.175 (astro-ph/0410085)

Ouyed, R., Dey, J., \& Dey, M. 2002, A\&A, 390, L39

Ouyed, R., Rapp, R., Vogt, C. 2005, ApJ, 632, 1001

Ouyed, R., Niebergal, B., Dobler, W., Leahy, D. 2006, ApJ, 653, 558

Paczynski, B., \& Haensel, P. 2005, MNRAS, 362, L4

Padmanabhan, T. Theoretical Astrophysics (Cambridge University Press, Cambridge, 2000)

Piran T., Shemi A., Narayan R., 1993, MNRAS, 263, 861

Rajagopal, K. \& Wilczek, F. 2000, in At the Frontier of Particle Physics/Handbook of QCD, Shifman, M. (ed.), World Scientific, vol. 3, 2061

Shovkovy, I. A., \& Ellis, P. J. 2002, Phys. Rev. C, 66, 015802

Usov, V. V. 1998, Phys. Rev. Lett., 80, 230

Usov, V. V. 2001, ApJ, 550, L179

Vogt, C., R., Rapp, \& R. Ouyed 2004, Nucl. Phys. A, 735, 543

Weber, F. 2005, Prog. Part. Nucl. Phys., 54, 193

Wang, X.Y., Dai, Z.G., Lu T. et al. 2000, A\&A, 357, 543

Witten, E. 1984, Phys. Rev. D, 30, 272

Xu, R. X., Qiao, G. J., \& Zhang, B. 1999, ApJ, 522, L109

Xu, R. X. 2002, ApJ, 570, L65

Xu, R. X. 2005, MNRAS, 356, 359

Xu, R. X. 2006, in: The proceedings of the 2005 Lake Hanas International Pulsar Symposium, Chin. Jour. A\&A Suppl., 6, 279

Yahil, A. 1983, ApJ, 265, 1047 
Yue, Y. L., Cui, X. H., Xu, R. X. 2006, ApJ, 649, L95

This preprint was prepared with the AAS LATEX macros v5.0. 


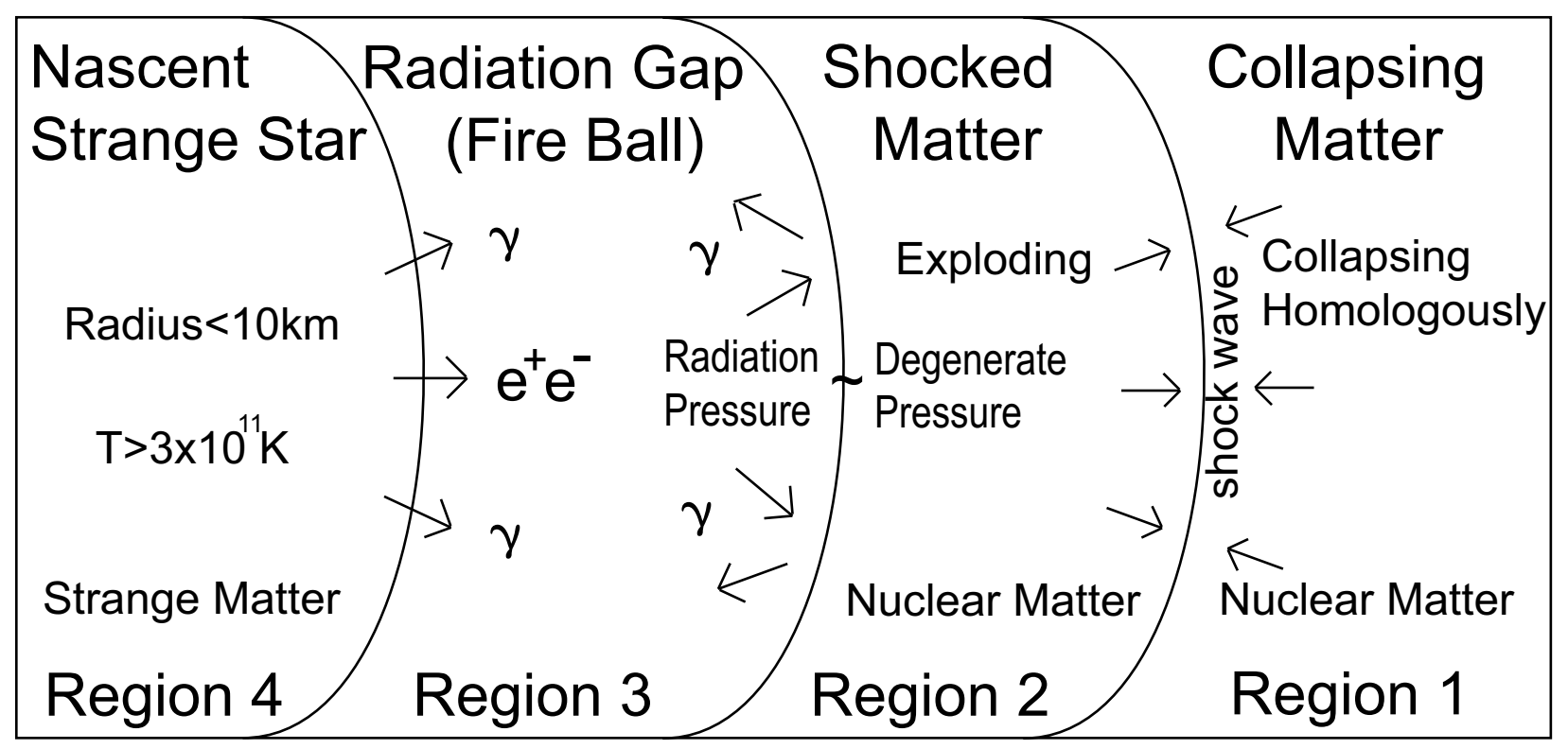

Fig. 1. - The outermost region 1 consists of the unshocked nuclear matter which is still infalling, assembled to the homologous solution (Goldreich \& Weber 1980; Yahil 1983). Behind the shock front which serves as the border and increases in thickness, region 2 comprises the shocked nuclear matter whose motion has been reversed by the shock. Between the nascent strange star in the center of the original collapsing core and region 2 is a fireball (region 3), a gap filled up with high energy photons and $e^{+} e^{-}$pair plasma, similar to the fireball in case of long-soft gamma-ray bursts. 


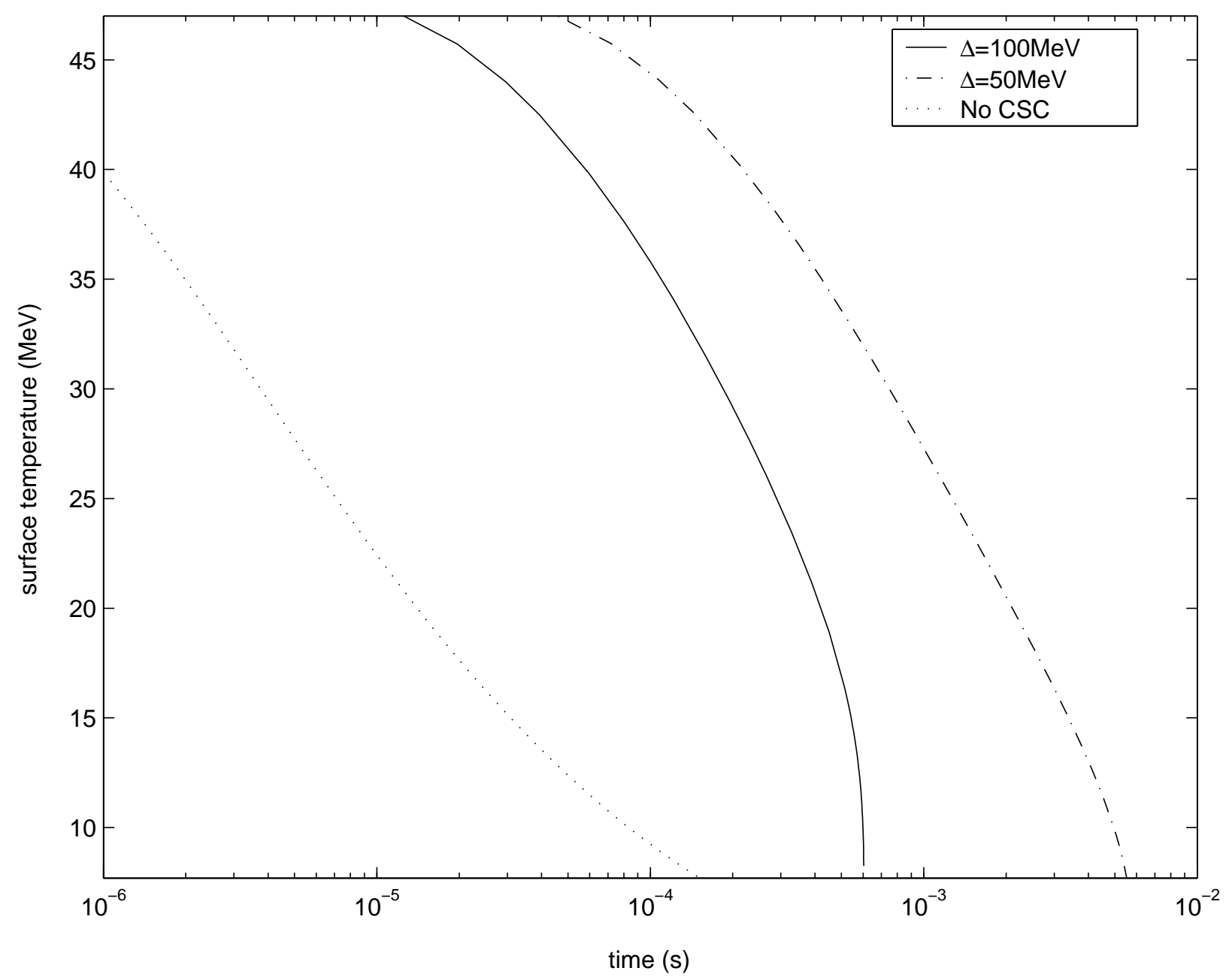

Fig. 2.- Cooling of a strange star with radius $R=9 \mathrm{~km}$ and initial temperature $T_{0}=50 \mathrm{MeV}$, for two different energy gaps of CFL matter, $\Delta=100 \mathrm{MeV}$ (solid line), $50 \mathrm{MeV}$ (solid-dotted line), and for normal quark matter (dotted line). The cooling of CFL matter is slower than normal quark matter, and the CFL matter cools faster with bigger energy gap. In about $\sim 1$ ms, the surface temperature of a CFL star will drop below $7.7 \mathrm{MeV}$, below which photon emission would not be effective. 


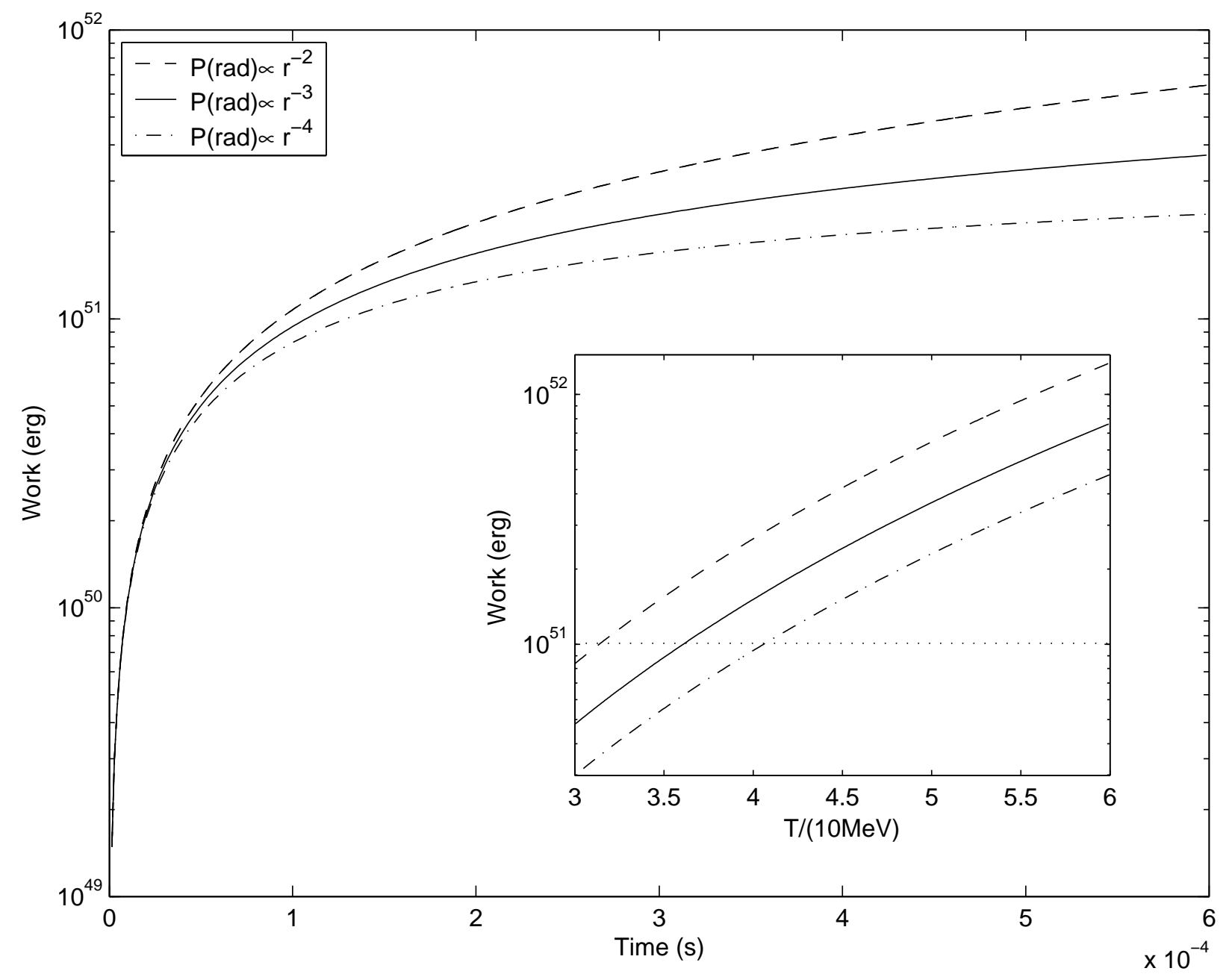

Fig. 3.- Work done by photon on the overlying mantle, with different radiation pressurefireball radius relationship and different initial temperatures. Both figures are under Strange Star model with radius $\mathrm{R}=10 \mathrm{~km}$. Different lines reflect different values of index $\mathrm{n}$ in Eq.(1). The larger figure shows the work increasing with time, which is calculated using initial temperature $T_{0}=50 \mathrm{MeV}$. The smaller figure at right-down corner shows the work with different initial temperatures, within radiation dominated timescale $t \sim 0.0006 s$. The work could exceed $10^{51} \mathrm{erg}$ if initial temperature is higher than about $40 \mathrm{MeV}$. 